\title{
Chronique des archives du Grand Ouest
}

\section{(2) OpenEdition \\ 1 Journals}

Édition électronique

URL : http://journals.openedition.org/abpo/1075

DOI : $10.4000 / a b p o .1075$

ISBN : 978-2-7535-1500-0

ISSN : 2108-6443

\section{Éditeur}

Presses universitaires de Rennes

\section{Édition imprimée}

Date de publication : 20 décembre 2005

Pagination : 229-254

ISBN : 978-2-7535-0243-7

ISSN : 0399-0826

\section{Référence électronique}

"Chronique des archives du Grand Ouest », Annales de Bretagne et des Pays de l'Ouest [En ligne], 112-4 | 2005, mis en ligne le 20 décembre 2005, consulté le 15 septembre 2020. URL : http:// journals.openedition.org/abpo/1075 


\title{
Chronique des archives du Grand Ouest
}

\author{
BRETAGNE \\ Archives départementales du Finistère
}

\author{
Fiche signalétique \\ Archives départementales deu Finistère \\ Cité administrative de Ty-Nay, Kerfeunteun - 29000 QUIMPER \\ Téléphone : 0298959191 - Télécopie : 0298953169 \\ Heures d'ouverture : lundi-vendredi 8 h $00-12$ h 00 et 13 h $30-17$ h 00 \\ Bâtiment annexe \\ Rue des Archives - 29200 BREST \\ Téléphone : 0298031614 - Télécopie : 0298476940 \\ Heures d'ouverture : lundi-vendredi $8 \mathrm{~h} 00-12 \mathrm{~h} 00$ et $13 \mathrm{~h} 30-17 \mathrm{~h} 00$
}

\section{1) Enrichissement des collections}

C'est dans le domaine des archives privées qu'il convient de signaler les entrées les plus significatives, dont :

Fonds Francis Gourvil (204 J) : archives personnelles de l'érudit morlaisien sur la toponymie et l'anthroponymie bretonnes, l'histoire de la Bretagne et des pays celtiques. Dépôt fait en janvier 2004 par les héritiers de Francis Gourvil.

Fonds des époux Fié-Fieux, manoir de Squividan (205 J) : archives personnelles du $\mathrm{xx}^{\mathrm{e}}$ siècle (comptables, médicales, correspondance). Don du Conseil général du Finistère (suite au legs du manoir et de ses meubles). Fonds en cours de classement, aucun instrument de recherche disponible.

Fonds famille Parquer (206 J) : archives personnelles et familiales, de 1661 à 1906. Don de M. Roger Moride en octobre 2004. Fonds classé doté d'un répertoire numérique détaillé dactylographié réalisé en février 2005. 
Il convient par ailleurs de souligner la volonté d'enrichissement du patrimoine archivistique qui a conduit le Conseil général à accorder aux Archives des crédits qui ont d'ores et déjà permis des acquisitions intéressantes sur l'abbaye de Daoulas par exemple, ou encore un carnet de dessins de Le Tendre, sur le Finistère et la Loire-Inférieure exécuté en 1895-1896. Ces documents seront prochainement inventoriés et mis à disposition du public.

Cette volonté se manifeste également dans la tentative de réintégration dans les fonds publics des archives de la manufacture des tabacs de Morlaix qui devrait connaître une issue favorable prochainement.

\section{2) Instruments de recherche et publications}

Les Archives départementales n'ont pas publié d'instruments de recherches depuis de longues années et préparent aujourd'hui la publication d'un répertoire de la série T qui a fait l'objet de travaux de classement importants ces derniers mois.

Une refonte des séries $\mathrm{J}$ et Fi est en cours. Ce travail en profondeur devrait laisser entrevoir une publication dans quelques petites années.

On signalera la mise en circulation en salle de lecture des outils suivants.

\section{Archives anciennes}

Sous-série 70 G - Paroisse de Gouézec, 17 articles, 1 p.

\section{Archives modernes}

Sous-série $7 \mathrm{M}$ - Agriculture, eaux et forêts, 64 articles, 14 p.

Sous-séries 4 Q-8 Q - Transcriptions hypothécaires, 7317 articles, 372 p.

Série T - projet de répertoire en cours d'achèvement.

\section{Archives contemporaines}

1397 W - Direction départementale des anciens combattants et victimes de guerre, 5 articles, $1 \mathrm{p}$.

$1428 \mathrm{~W}$ - Office national des forêts - service interdépartemental de Quimper, 23 articles, 2 p.

\section{Archives privées}

$134 \mathrm{~J}$ - Euvre nationale des orphelins et orphelines de la guerre de 1870-1871 - Sous-comité de Quimper, 2 p.

209 J - Fonds Gourvil, 199 articles, 14 p.

Sous-série V dépôt - Paroisse de Gouézec, 25 articles, 2 p. 


\section{Archives notariales}

4 E 92-98, 100, 101 (Guipavas, Landerneau, Landivisiau), 998 articles, $57 \mathrm{p}$.

\section{3) Échos et nouvelles}

Le Conseil général a adopté en réunion ordinaire de juin 2005 un plan de développement des Archives se déclinant en 4 objectifs stratégiques, 10 axes prioritaires et 34 actions, chacune comportant d'ailleurs plusieurs facettes ou " opérations".

Le premier objectif consiste à démocratiser l'accès aux archives en renouvelant l'offre au public finistérien et extérieur. Ceci implique de recourir aux nouvelles technologies, de mettre en place un réseau territorial de consultation et des actions pédagogiques et culturelles et de diffuser des instruments de recherche.

Le second objectif touche la production et la gestion de l'information administrative contemporaine, domaine où il convient de relever le défi de l'archivage électronique, d'améliorer et de développer des systèmes de préarchivage classiques et la prise en charge des versements en dématérialisant la procédure par exemple.

En troisième lieu le plan cherche à faire des propositions tendant à aménager des territoires et à développer des réseaux. Les communes et l'intercommunalité sont à cet égard des partenaires déterminants, même s'il faut aussi développer des actions avec les partenaires culturels et associatifs, accompagner et soutenir la recherche fondamentale, avec le souci permanent de mieux faire connaître et faire comprendre les archives.

Enfin et quatrièmement il s'agit de renforcer l'action et de changer l'image des Archives dans le Finistère, de développer des outils de veille et d'expertise et la normalisation de la gestion des archives, sans oublier d'organiser les Archives et d'affirmer leur rôle et leur place.

La réalisation de ce plan devrait prendre plusieurs longues années au cours desquelles les dépôts finistériens de Brest et de Quimper devraient faire l'objet d'études d'extension.

Une exposition pédagogique itinérante sur la loi de séparation des églises et de l'État dans le Finistère sera présentée en décembre 2005 accompagnée d'un document d'exploitation pédagogique.

Le cadastre napoléonien a été numérisé et mis à disposition sous cette forme dans les salles de lecture de Brest et de Quimper. Les Archives du Finistère continuent bien entendu la numérisation des registres paroissiaux et d'état civil... 


\section{Archives départementales d'Ille-et-Vilaine}

Fiche signalétique
Archives départementales d'Ille-et-Vilaine
20 avenue Jules-Ferry - 35700 RENNES
Téléphone : 0299272150 - Télécopie : 0299272151
e-mail : archives@cg35.fr
Heures d'ouvertures : lundi-vendredi 8 h 30 à $17 \mathrm{~h} 30$
fermeture annuelle du 1er au 15 août
Pour une première approche des fonds, consulter : Guide des Archives d'Ille-et-
Vilaine, Tome I, volume 1. Archives départementales 1re partie [ne comprend pas
la période de 1790 à nos jours] et archives hospitalières, Rennes, 1994, $710 \mathrm{p}$;
tome II, Archives communales, Rennes, 1991, $512 \mathrm{p}$.

\section{1) Enrichissement des collections}

\section{Archives publiques}

Les Archives départementales d'Ille-et-Vilaine ont reçu en 2004515 m linéaires de documents provenant essentiellement des services déconcentrés de l'État. On relèvera particulièrement les versements :

- de la justice, comprenant essentiellement les jugements et procédures du tribunal de grande instance de Rennes (1968-1996), et de la justice administrative (217 m linéaires),

- de l'INSEE (57 m linéaires), comprenant l'échantillon du recensement de population de 1999,

- de la préfecture (40 m linéaires), concernant les enquêtes publiques en matière d'aménagement et d'environnement (1977-2000) et les dossiers administratifs des installations industrielles classées fermées (1940-1976),

- des dossiers de détenues libérées entre 1978 et 1992 par le Centre pénitentiaire de Rennes (69 m linéaires),

- du tribunal de commerce de Saint-Malo (21 m linéaires),

- du commissariat de Saint-Malo (10 m linéaires),

Les documents entrés antérieurs à 1940 sont des minutes notariales (30 m linéaires), les fonds de subdivisions de l'Équipement (55 m linéaires) et les archives d'Ancien Régime des paroisses de Brie et Cancale.

\section{Archives privées}

On signalera les archives de Pierre Le Treut, ancien conseiller général et régional (25 m linéaires), le fonds Le Loroux, négociants en toiles à Rennes au XIX ${ }^{\mathrm{e}}$ siècle (153 J, 3,4 m linéaires), les compléments du fonds Louise, entrepreneur rennais du $\mathrm{XIX}^{\mathrm{e}}$ siècle, et du fonds du comité d'études et de liaison des intérêts bretons (CELIB), les archives de la section départementale de la MRIFEN (152 J, 1,5 m linéaires) et du centre régional d'études et d'action sur les inadaptations et les handicaps (CREAI de Bretagne) (155 J, 25,5 m linéaires), ainsi qu'environ 150 travaux universitaires. 


\title{
2) Instruments de recherche
}

On citera particulièrement ceux qui décrivent le supplément de la soussérie $1 \mathrm{G}$ (archives du diocèse de Rennes sous l'Ancien Régime remises par l'évêché), les fonds des fédérations nationale et départementale des CUMA $(127 \mathrm{~J}$ et $128 \mathrm{~J})$, le fonds Guy Parigot (150 J), complémentaire de celui de la Comédie de l'Ouest, le fonds Guy Verdoïa, architecte $(97 \mathrm{~J})$, le fonds de la conservation départementale des antiquités et objets d'art (161 J), la série K (publications officielles, 1800-1940), la sous-série $13 \mathrm{~T}$ (procès-verbaux du baccalauréat de 1880 à 1940).

Pour les archives contemporaines (après 1940), outre la mise à disposition de la quasi totalité des versements entrés en 2004, on peut citer les travaux de reprise sur les fonds du Génie rural ( $241 \mathrm{~W}, 522 \mathrm{~W})$, le fonds des rapatriés d'Algérie $(161 \mathrm{~W})$ provenant du cabinet du préfet et les dossiers d'étrangers entrés en France de 1940 (quelques dossiers antérieurs) à 1970 provenant de la préfecture ( $265 \mathrm{~W}$ ), ainsi qu'une analyse détaillée des versements des succursales de la Banque de France (1-4 ETP).

Par ailleurs, un petit guide de recherche a été élaboré sur les sources de l'histoire de la dernière guerre aux Archives d'Ille-et-Vilaine.

Enfin, on notera les classements des archives communales d'Argentrédu-Plessis, Baulon, Brécé, Châteaugiron (communauté de communes), Châteauneuf, La Gouesnière, Guignen, Irodouër, Lassy, Nouvoitou, SaintErblon, Saint-M'Hervé et Treffendel.

\section{3) Échos et nouvelles}

- Numérisation des cartes et plans de la série C (fonds de l'intendance et des États de Bretagne). Opération achevée au début 2005.

- Poursuite de la réflexion engagée sur l'archivage électronique, en lien avec la direction des Archives de France : les Archives d'Ille-et-Vilaine sont l'un des services départementaux pilotes en la matière.

- Poursuite de la préparation du déménagement du service (nombreuses opérations de (re)conditionnement).

\section{Archives municipales de Rennes}

\author{
Fiche signalétique
}

Archives municipales de Rennes

18, avenue Jules-Ferry, B.P. 3126 - 35031 RenNes Cedex

Téléphone : 0299380347 - Télécopie : 0299360745

e-mail : archives@ville-rennes.fr

site internet : www.archives.rennes.fr

Heures d'ouvertures : lundi-vendredi 9 h 00-12 h 00 et 13h 00-17 h 00 


\section{1) Enrichissement des collections}

\section{Archives publiques}

Nous avons reçu cette année $300 \mathrm{ml}$ d'archives provenant des services municipaux les plus divers. Nous signalerons spécialement les dossiers concernant les travaux dans les écoles (1974-2001), un versement du musée des Beaux-Arts concernant les expositions et les acquisitions d'œuvres (1977-2000) et, en matière d'urbanisme, les dossiers du POS (2001) et du PLU (2003).

\section{Archives privées}

Les Archives de Rennes se sont enrichies de trois principaux fonds privés : celui de l'Orchestre de Bretagne (1988-1998), du patronage de La Tour d'Auvergne (1897-1997) et des Amitiés sociales (1955-2001).

\section{2) Instruments de recherche et publications}

Les versements contemporains (W) sont traités le plus rapidement possible et sont alors saisis dans notre base de données et indexés. Ainsi cette année, $217 \mathrm{ml}$ ont été traités.

\section{3) Échos et nouvelles}

Notre site Internet a pris son régime de croisière : il est très consulté (en moyenne 250 connexions par jour). En 2004, plus de 1450000 pages de documents numérisés ont été consultées. Ceci est bien sûr sans commune mesure avec nos statistiques de la salle de lecture qui restent quasiment stables cependant (1 019 lecteurs en 2004 contre 1025 en 2003; 6844 dossiers papiers contre 6903; les documents numérisés consultés en salle de lecture ne sont pas comptabilisés).

Les conférences mensuelles données dans le cadre de l'Histoire à l'heure des archives ont donné lieu presque à chaque fois à une présentation de documents.

Par ailleurs, deux expositions sur panneaux ont été réalisées et présentées dans différents lieux, l'une intitulée "Louis Chouinard, architecte de Rennes à Bruz " et l'autre "La fête de la jeunesse : 75 ans et toujours jeune ". 


\section{Archives départementales du Morbihan}

Fiche signalétique
Archives départementales du Morbihan
80 rue des Vénètes - 56010 VANNES CEDEX
Téléphone : 0297463252 - Télécopie : 0297464876
e-mail : Cg56.Archives@wanadoo.fr
site internet : www.cg56.fr
Heures d'ouvertures : lundi-vendredi $9 \mathrm{~h} 00-17 \mathrm{~h} 30$
samedi $9 \mathrm{~h} 00-12 \mathrm{~h} 00$
fermeture mensuelle $:$ le $2^{\mathrm{e}}$ mercredi matin de chaque mois
fermeture annuelle $:$ du $1^{\text {er }}$ au 15 septembre

\section{1) Enrichissement des collections}

\section{Archives publiques}

Les versements et dépôts d'archives publiques pour l'année 2004 représentent 375 m linéaires, en provenance notamment des services déconcentrés de l'État : Direction départementale de l'agriculture et de la forêt, Direction départementale de l'équipement, tribunaux, Trésor public, Direction départementale de la sécurité publique (commissariats de police de Lorient et Pontivy), INSEE, Centre régional opérationnel de surveillance et de sauvetage atlantique (CROSSA Étel).

L'effort particulier entrepris depuis deux ans pour mener à bien la collecte des arriérés d'archives publiques a abouti au versement ou au dépôt, en 2004, de deux fonds d'études notariales (25 m linéaires), des archives de l'Établissement public de santé mentale de Saint-Avé (70 m linéaires), ainsi que des archives centenaires de l'état civil (fonds des greffes des tribunaux de grande instance de Vannes et de Lorient, $37 \mathrm{~m}$ linéaires).

Citons enfin, au titre des archives communales déposées, l'entrée aux Archives du fonds de la commune de Malestroit (3,50 m linéaires).

\section{2) Instruments de recherche et publications}

Les travaux de classement ont porté principalement sur les archives modernes et contemporaines, et une attention toute spéciale a été confiée à la rédaction d'instruments de recherche normalisés, dans le but de permettre leur consultation future par l'intermédiaire du site Internet des Archives. Ont été classés ou reclassés en particulier les minutes et répertoires de notaires (près de 30 m linéaires, dont les remarquables archives de l'étude de Mes Le Touze et Brisset à Lorient, qui remontent au XVIII siècle et sont riches d'enseignements pour l'histoire de la compagnie des Indes et du commerce maritime lorientais durant cette époque), les dossiers d'administration communale produits par la Préfecture entre 1800 et 1940 (14 communes ont été reclassées) l'état civil du XIX siècle (186 m linéaires). 
À signaler également l'inventaire des archives du Centre régional opérationnel de surveillance et de sauvetage atlantique (CROSSA Étel) couvrant la période 1958-1998, et représentant $37 \mathrm{~m}$ linéaires, celui des archives de l'INSEE (38 m linéaires, recensements de 1954 à 1990), du commissariat de police de Lorient (45 m linéaires, 1941-1997), et de la Banque de France (succursales de Vannes, Lorient et Pontivy pour la période 1803-1985). Enfin ont été menés à bien les inventaires des archives des communes des Forges, de Saint-Caradec-Trégomel et de Saint-Laurent-sur-Oust.

\section{3) Échos et nouvelles}

Durant l'année 2004, 3920 lecteurs ont fréquenté la salle de lecture des Archives et ont consulté 36950 documents (liasses, registres ou cartons d'archives). Il est à noter que l'état civil ancien et moderne n'est pas inclus dans ces statistiques, la majeure partie des documents ayant été microfilmée et/ou numérisée et étant consultée sur écran (lecteur de microfilm ou ordinateur).

D'une manière générale les conditions d'accueil des lecteurs ont été considérablement améliorées durant l'année 2004, par l'ouverture au public d'un nouvel espace de consultation, réservé pour l'instant aux lecteurs de microfilms, et qui sera à terme uniquement occupé par des ordinateurs. Le parc informatique réservé au public est passé de 5 machines en 2003 à 16 machines pour 2004, permettant la consultation de la presse ancienne et de l'état civil numérisés du département dans d'excellentes conditions.

La politique de numérisation entreprise depuis quelques années s'est poursuivie en 2004 et a donné lieu à la réalisation de 470000 vues numérisées concernant la presse ancienne morbihannaise (fin de l'opération) et l'état civil du XIX ${ }^{\mathrm{e}}$ siècle. De même deux fonds d'archives privées regroupant 790 plaques de verre ont été numérisés en 2004, et l'un d'entre eux sera présenté au public dans le cadre d'une exposition en 2005. Ces vues seront installées en 2005 sur le serveur des Archives et consultables en ligne en salle de lecture.

Enfin les Archives départementales ont entamé en 2004 une politique de réflexion sur le développement d'un moteur de recherche destiné à l'interrogation en ligne des instruments de recherche normalisés produits par le service. L'outil, mis au point par une équipe d'étudiants en DESS informatique de l'Université de Bretagne Sud, est en test en salle de lecture depuis le mois de septembre 2004 et sera présenté à la communauté archivistique et informatique de l'interrégion Bretagne-Pays de la Loire le 7 avril 2005.

Dans le domaine de l'animation culturelle, les Archives départementales ont préparé ou accueilli quatre expositions au cours de l'année écoulée :

- "Les archives au grand large : patrimoine écrit et modélisme naval " (du 19 janvier au 20 mars 2004); cette exposition a été complétée par deux conférences, "La découverte des Amériques à travers La Belle " et "La voile au travail". 
- « Mai des arts : lumières et transparences » : cette manifestation a permis de présenter, dans le hall d'accueil des Archives départementales, les productions artistiques des établissements scolaires du premier et du second degré de l'enseignement public et privé du département; elle a été l'occasion d'établir un partenariat privilégié avec l'Inspection académique du Morbihan, qui devrait se poursuivre dans les années à venir.

- "Yves Guillou, architecte d'une nouvelle Bretagne" (du 4 juin au 2 octobre 2004); cette exposition, réalisée en partenariat avec le Conseil en Architecture, Urbanisme et Environnement du Morbihan (CAUE) a permis de valoriser le fonds d'archives exceptionnel (96 m linéaires, couvrant la période 1937-1994) remis en don officiellement aux Archives départementales en 2004 par cet architecte prestigieux, et dont l'inventaire informatisé est d'ores et déjà consultable par le public.

- «Le Morbihan entre deux guerres »; élaborée en collaboration étroite avec les professeurs relais du service éducatif des archives, l'exposition, qui a débuté le 4 novembre 2004 et sera visible jusqu'au 30 avril 2005, est accompagnée d'une brochure pédagogique illustrée et commentée d'une soixantaine de pages, ainsi que d'une collection de six cartes postales représentant des affiches politiques de l'entre-deux-guerres.

Ces quatre expositions, qui ont mobilisé largement le personnel des Archives, particulièrement le pôle conservation et la cellule d'action culturelle et éducative, ont réuni plus de 3000 visiteurs au cours de l'année 2004.

\section{PAYS DE LA LOIRE \\ Archives départementales de Loire-Atlantique}

\begin{tabular}{|c|} 
Fiche signalétique \\
Archives départementales de Loire-Atlantique \\
6 rue de Bouillé, B.P. 23505 - 44035 NANTES CEDEX 1 \\
Téléphone : 0251 729320 - Télécopie : 02 40202691 \\
e-mail : archives@cg44.fr \\
site internet : http://culture.cg44.fr \\
Heures d'ouvertures : lundi-vendredi 8h30-12 h 15 et 13h $30-17 \mathrm{~h} 15$ sur réservation \\
Consultation des documents numérisés : 44 rue de Strasbourg \\
(réservation au 0240997811) \\
Consultation des documents originaux : 1 rue E.-Tessier \\
(réservation au 0251 729899) \\
Fermeture annuelle la première quinzaine de juillet \\
Pour une première approche des fonds, consulter : \\
Guide des archives, Nantes, 1962-1964, 2 vol.
\end{tabular}




\section{1) Enrichissement des collections}

\section{Archives publiques}

Les entrées de documents publics aux Archives départementales de Loire-Atlantique en 2004 ont été de 372 mètres linéaires. Parmi ces versements, on peut noter les suivants :

- Conseil général : Cabinet du président : direction de la communication, organisation de manifestations, diapositives, discours (1988-1999); Direction de l'aménagement et de l'environnement : service des transports (1981-2000), service de l'équipement des collectivités locales et du cadre de vie (1987-1998); Direction du patrimoine immobilier : service bâtiments et patrimoine (1976-1996), service programmation et travaux dans les collèges (1983-1994); Direction du développement culturel : développement éducatif et recherche (1973-1998); Association départementale de sauvegarde de l'enfance et de l'adolescence : dossiers de jeunes confiés entre 1911 et 1980.

- Préfecture : Direction de la réglementation et de l'administration générale : listes électorales, élections diverses, affaires militaires, dons et legs (1971-2000).

- Direction régionale des affaires culturelles : archives de la coordination et des conseillers sectoriels (1953-2000); Direction régionale de l'industrie, de la recherche et de l'environnement : contrôle des véhicules, dossiers de réception par type donnant des renseignements techniques sur la construction automobile (1985-1995); Direction départementale du travail, de l'emploi et de la formation professionnelle : contrôle des établissements de plus de 50 salariés (1990-1999); Direction départementale de la sécurité publique : dossiers des individus surveillés (1910-1950); Inspection académique : dossiers de carrière.

- Autorités judiciaires : Tribunal d'instance de Saint-Nazaire, annexe du greffe de Savenay : rapports d'expertise, minutes de jugements, dossiers de procédure, plumitifs d'audience (1969-1998), greffes des justices de paix du Croisic, de Savenay, d'Herbignac et de Guérande : registres d'audience, minutes de jugements, dossiers de procédure, contrats d'apprentissage, rapports d'expertise (1938-1993); Tribunal de grande instance de Saint-Nazaire : minutes de jugements, dossiers de procédure, registres d'audiences, répertoires de notaires (1937-1998); Conseil de prud'hommes de Nantes : procès-verbaux des assemblées générales, accords collectifs, règlements intérieurs, minutes des décisions, registres d'audience, dossiers de procédure (1930-1998); Cour administrative d'appel : minutes des ordonnances et audiences, dossiers de procédure, rôles des audiences (1996-1998); Chambre régionale des comptes : comptes de gestion des exercices 1983 à 1992; Centre pénitentiaire de Nantes : dossiers individuels des détenus libérés, registres d'écrou, registres de main courante (1985-1993).

- Port autonome de Nantes-Saint-Nazaire : dossiers des réseaux d'alimentation en eau potable et d'assainissement, de travaux de défense contre 
la mer, de construction et de travaux d'entretien des ports de plaisance et de pêche (1932-1993).

- Notaires : compléments de versements d'études de Campbon, Nantes, Pornic, Saint-Julien-de-Concelles.

- Communes : dépôt des archives modernes des communes de La Chapelle-sur-Erdre et Saint-Sébastien-sur-Loire.

\section{Archives privées}

Les fonds et collections d'origine privée se sont accrus notamment de deux fonds d'architecte : Atelier 44/Ferronière et $\mathrm{H}$. Leroy, de plans et photographies concernant les Ateliers et chantiers de Bretagne, d'une charte signée d'Anne de Bretagne datée de 1489, de documents relatifs à l'armement nantais P. Ciret, François aîné et Baudot-Ducarrey, particulièrement pour les affaires de la compagnie de Venezuela de Nantes (1836-1846).

\section{Archives iconographiques et audiovisuelles}

Les fonds iconographiques se sont notablement enrichis par achat, don et dépôt (cartes et plans, cartes postales de Nantes et du département, photographies, notamment de Nantes sous les bombardements, plaques de verre), et la convention avec la cinémathèque de Bretagne a permis le dépôt de 260 films et cassettes vidéo.

\section{2) Instruments de recherche et publications}

\section{Instruments de recherche publiés}

Archives de la Seconde Guerre mondiale. Tome III : services de l'État et organismes de temps de guerre (1940-1946).

Archives des sous-préfectures (1830-1946).

Hôpitaux de Loire-Atlantique des origines à 1940 (à l'exception des hôpitaux nantais).

\section{Instruments de recherches dactylographiés}

À noter, parmi les instruments de recherche mis en service :

Sous-série $3 \mathrm{Q}$ : le reclassement d'un certain nombre de bureaux de l'enregistrement a donné lieu à 137 pages d'inventaire.

Les fichiers manuels des séries modernes ont été repris sous forme de répertoires dactylographiés (890 pages).

\section{Autre publication}

Pour en finir avec Anne de Bretagne? Actes de la journée d'études organisée aux Archives départementales de Loire-Atlantique le 25 mars 2002. 


\section{Bibliothèque}

mise en place d'un logiciel informatisé pour la création des notices bibliographiques et la consultation du catalogue des ouvrages de la bibliothèque historique.

\section{Bases de données}

Les bases se sont enrichies de 4099 notices pour les archives contemporaines et de 3800 pour les fonds iconographiques.

\section{3) Échos et nouvelles}

Avant la fermeture au public, a été présentée une exposition, accompagnée de conférences et d'une projection de la cinémathèque de Bretagne, sur le thème de l'architecture balnéaire.

La signature officielle du contrat de dépôt des archives de la famille de Sesmaisons (185 J) a donné lieu à une exposition aux Archives. Ce fonds de famille, qui représente 23 m linéaires de documents privés, s'échelonne sur sept siècles, l'acte le plus ancien datant de 1219. Pour l'Ancien Régime, les archives déposées ont trait aux membres de cette importante famille du comté nantais, mais aussi à des familles alliées fixées dans la presqu'île guérandaise. S'y trouvent également les titres d'autres familles, dont les biens sont situés en Seine-Maritime, dans les Côtes-d'Armor et dans la Manche, entrés aussi par alliance. Pour le XIX siècle, les documents concernent les possessions récentes de la famille de Sesmaisons, principalement le domaine de la Desnerie sur la commune de La Chapelle-sur-Erdre. Deux membres de cette famille y sont particulièrement bien représentés : LouisOlivier (1807-1874), conseiller général, l'un des chefs du parti légitimiste et engagé dans le développement agricole du département; Claude-MarieRogatien (1835-1920), qui fit une belle carrière militaire. Il servit d'abord à Alger, participa à la guerre de 1870-1871, fut attaché militaire à l'ambassade de France à Berlin, sous-chef au ministère de la guerre, puis général de brigade. Il devint également conseiller général du département. Il fut fait grand officier de la Légion d'honneur et reçut plusieurs décorations étrangères.

Outre les expositions réalisées sur place, les Archives départementales ont participé à d'autres par le prêt de 85 documents, notamment Nantes et les cap-horniers, à la maison des Hommes et des Techniques, Des moteurs et des ailes, au lycée Clemenceau, et ont apporté leur concours à la réalisation de la manifestation commémorant le bicentenaire de la première remise de la Légion d'honneur dans le département.

Le service éducatif a accueilli 1200 scolaires pour la participation aux divers ateliers proposés et a mis au point deux nouveaux documents pédagogiques : Traverser l'Erdre, hier et aujourd'hui, et Sceaux du Moyen Âge.

La numérisation des registres d'état civil, à l'exception de ceux de Nantes, a été achevée, celle de registres de délibérations de 14 communes (10080 vues), de cartes postales (15000 vues), de matricules militaires des 
conscrits de Loire-Atlantique entre 1878 et 1980 (41000 vues) a été réalisée. L'accès à ces documents numérisés est possible non seulement dans la salle de lecture multimédia des Archives, mais aussi à la Maison du département ouverte à Ancenis.

En prévision de l'ouverture du chantier de restructuration et d'extension des Archives départementales, qui doit durer 30 mois, la salle de lecture du site a été fermée en avril 2004. Deux salles provisoires de consultation ont été ouvertes à l'extérieur : une pour la consultation des documents originaux et une pour celle des documents numérisés (voir fiche signalétique).

\section{Archives municipales de Nantes}

\begin{tabular}{|c|}
\hline Fiche signalétique \\
Archives municipales de Nantes \\
1, rue d'Enfer - 44094 NANTES CEDEX 01 \\
Téléphone : 0240419585 - Télécopie : 0240473879 \\
e-mail : archives@mairie-nantes.fr \\
site internet : www.archives.nantes.fr \\
Heures d'ouvertures : lundi-vendredi 8 h $30-12 \mathrm{~h} 30$ et $13 \mathrm{~h} 30-18 \mathrm{~h} 00$ \\
Pour une première approche des fonds, consulter : \\
Guide des Archives, par Christine Nougaret, Nantes, 1991, 156 p.
\end{tabular}

\section{1) Enrichissement des collections}

\section{Archives publiques}

La complète saturation des locaux ne permet plus aux Archives de remplir la mission première de collecte auprès des services municipaux.

Ces derniers ne peuvent plus procéder aux versements des documents contemporains.

En 2004, outre les registres d'état civil de 1904 de Nantes, Chantenay et Doulon, sont entrées les archives de la construction de la Cité des Congrès (marchés, plans, photographies).

\section{Archives privées}

Madame Pressensé a fait don aux Archives de 193 plaques de verre. Ces photographies illustrant Nantes, la Loire-Inférieure et la Bretagne ont été prises par son grand-père Paul Fleury fin XIX

Les Archives municipales ont fait l'acquisition de dix plans de l'architecte Portail, plans du XVIII ${ }^{\mathrm{e}}$ siècle. 


\section{2) Instruments de recherche et publications}

Le fonds de la commune de Chantenay (2 Z) est presque entièrement classé et a fait l'objet d'un inventaire provisoire.

Dans la sous-série $4 \mathrm{M}$, tous les dossiers des bâtiments à usage culturel (théâtres, musées...) sont classés, décrits, indexés et saisis dans la base Archives.

Actuellement sont accessibles directement à partir de la base environ 7000 photographies, 1746 cartes postales, 2111 plans et 409 affiches.

\section{3) Échos et nouvelles}

En 2004, le site Internet a reçu 46801 visiteurs, soit une moyenne de 3900 par mois.

En septembre 2004, à l'occasion du centenaire de la mairie de Chantenay, les Archives municipales ont présenté l'exposition : Le Chantenay de Griveaud, 1900-1908.

\section{Archives municipales d'Angers}

\begin{tabular}{|c|}
\hline Fiche signalétique \\
Archives municipales d'Angers \\
Hôtel de ville, BP 3527 - 49035 ANGERS CEDEX 01 \\
Téléphone : 0241054281 - Télécopie : 0241053911 \\
e-mail : sylvain.bertoldi@angers.fr \\
Heures d'ouvertures : lundi-vendredi $14 \mathrm{~h} 00-17 \mathrm{~h} 00$ \\
\hline
\end{tabular}

\section{1) Enrichissement des collections}

Le service a reçu 197 mètres de documents.

\section{Services municipaux}

- Santé publique : écologie urbaine. « Casier sanitaire », environnement, plaintes pour nuisances, demandes d'aménagement (1967-1993),

- urbanisme : modification du plan d'occupation des sols (1982-1994); permis de construire (1965-1971); déclarations de travaux (2001-2002),

- bâtiments : travaux (1980-2004),

- prospective - évaluation - qualité : " écoute active des Angevins ", amélioration de l'accueil et des services,

- musées : budgets, correspondance, expositions, projets culturels (1920-1995). 


\section{Archives privées}

- Commerces angevins : publicités, factures à en-tête, catalogues, bons, chromolithographies, objets publicitaires, etc.,

- Saboterie et magasin de chaussures Lebreton, rue Baudrière : mandats, factures, etc.,

- Studio Bruel, photographie : portraits (1968-1983)

- Hôtel de la Boule d'Or, boulevard Carnot : livre d'or

- Mutualité angevine du gaz : drapeau (début xx siècle)

- Foyer symphonique angevin : fanion (1932)

- Belle-Beille : reportage sur la construction du quartier, par l'architecte Jean Pattin.

\section{Archives iconographiques}

9945 nouvelles pièces (cartes postales, photographies, diapositives, numérisations...).

Enrichissement des fonds photographiques par numérisation de documents originaux provenant de collections privées.

\section{2) Publications - Internet}

- Chronique historique mensuelle dans Vivre à Angers.

- Portail historique d'Angers sur le site internet de la ville [www.angers.fr/archives]. Il a reçu 63285 visites en 2004.

\section{3) Échos et nouvelles}

\section{Exposition}

Le service a présenté, salle Chemellier, une exposition d'été (28 mai22 août) consacrée aux commerces d'Angers dans l'histoire ( $\mathrm{XIX}^{\mathrm{e}}-\mathrm{xX} \mathrm{e}^{\mathrm{e}}$ siècles) : $A u$ Bonheur des Angevins. Elle a suscité un grand intérêt chez les Angevins, mais aussi chez les touristes de passage (plus de 22000 visiteurs) et permis au patrimoine municipal de s'enrichir de nombreux documents.

Une première partie présentait les sources sur l'histoire du commerce et les différents types de commerces qui ont existé à Angers du XVIII ${ }^{\mathrm{e}}$ siècle à nos jours. Six magasins reconstituaient ensuite une rue commerçante, aux alentours de 1900-1930.

La manifestation a été l'occasion d'une fructueuse collaboration avec commerçants, collectionneurs, antiquaires, archives départementales et musées, avec la Chambre de commerce et d'industrie et l'Agence d'urbanisme de la région angevine qui a dessiné d'après les bases de données des archives la géographie commerciale au début du $\mathrm{xx}^{\mathrm{e}}$ siècle. 


\section{Informatique - Numérisation}

Numérisation de 2300 clichés de la collection iconographique Robert Brisset, de 500 affiches et des délibérations du conseil municipal (19701980).

Une chronique régulière est parue parallèlement pendant six mois dans Le Courrier de l'Ouest, "Petite histoire des grandes enseignes angevines ", rédigée en alternance avec le journaliste Yvan Duvivier.

\section{Activités documentaires}

- 4790 recherches et sélections documentaires effectuées,

- 854 abonnements gérés,

- Revue de presse quotidienne.

\section{Archives municipales de Saumur}

\begin{tabular}{|c|}
\hline Fiche signalétique \\
Archives municipales de Saumur \\
Adresse topographique : 25 quai Carnot (entrée annexe : rue du Pavillon) \\
Adresse postale : Hôtel de Ville, BP $300-49408$ SAUMUR CEDEX \\
Téléphone : 0241402870 - Télécopie : 0241402879 \\
e-mail : archives@ville-saumur.fr \\
Responsable du service : Véronique Flandrin \\
Heures d'ouvertures : lundi-vendredi $13 \mathrm{~h} 30$ à $17 \mathrm{~h} 15$ \\
\hline
\end{tabular}

\section{1) Enrichissement des collections}

Trois fonds privés sont venus enrichir les Archives de la ville de Saumur cette année :

- La collection G. Perrusson comprenant 284 plaques de verre photographiques (négatifs aux formats variés : 18x24, 24x30 et 30x40 cm). Vers 1950 , ce photographe prend la succession de M. Lecomte, dont le studio est installé au $n^{\circ} 57$ de la rue d'Orléans à Saumur. Le fonds photographique comprend la production de Toussaint Le Roch, installé à Saumur en 1862, celle de son employé et successeur, Victor Coué, et du repreneur de l'affaire en 1914, M. David. La consultation de la version numérique de la collection, cotée $34 \mathrm{Fi}$, sera possible avant la fin de l'année 2005.

- Le fonds d'archives de Louis Boivin et de sa fille Marguerite, respectivement docteur en médecine (1900-1950) et assistante sociale (1940-1990). Répertorié sous la cote $34 \mathrm{Z}$, ce fonds contient les papiers et les diplômes des deux personnages, de nombreuses photographies concernant essentiellement les membres de la famille, mais également la ville de Saumur pendant la Seconde Guerre mondiale. Enfin, les notes, les rapports de stages 
et les exposés de Marguerite nous éclairent sur l'enseignement délivré aux futures assistantes sociales dans les années 1940.

- Le fonds d'archives de la plus ancienne loge maçonnique de Saumur, "La Persévérance ", fondée en 1745, comprend les registres des procèsverbaux, les tableaux des membres de la loge, la comptabilité, et quelques documents figurés.

\section{2) Instruments de recherche}

La sous-série $1 \mathrm{O}$ intitulée "Voirie et réseaux divers " ( $\mathrm{XIX}^{\mathrm{e}}-\mathrm{xx}^{\mathrm{e}}$ siècle) et la sous-série $2 \mathrm{H}$ intitulée "Administration militaire " ( $\mathrm{XIX}^{\mathrm{e}}-\mathrm{XX}^{\mathrm{e}}$ siècle) ont fait l'objet d'inventaires détaillés. Ils sont, bien sûr, accessibles en salle de lecture.

\section{3) Échos et nouvelles}

Le programme de numérisation se poursuit. Il concerne, cette année, les registres d'état civil des communes de Bagneux et de Dampierre, une partie de la collection du journal L'Écho Saumurois (1853-1872), et les 284 plaques photographiques de la collection Perrusson.

Enfin, dans le cadre des Journées du Patrimoine 2005, les Archives municipales feront la présentation de la production documentaire d'un architecte emblématique de la ville de Saumur, Charles Joly-Leterme (1805-1885).

\section{Archives municipales de Laval}

Fiche signalétique
Archives municipales de Laval
37 quai Paul-Boudet, BP 1327 - 53013 LAVAL CEDEX
Téléphone : 0243493684 - Télécopie : 02 4356 1372
e-mail : archives@mairie-laval.fr
site internet : www.mairie-laval.fr/Franc/archives/index.php
Heures d'ouvertures : mardi $14 \mathrm{~h} 00-17 \mathrm{~h} 00$
mercredi $9 \mathrm{~h} 00-12 \mathrm{~h} 00,14 \mathrm{~h} 00-17 \mathrm{~h} 00$
jeudi, vendredi $14 \mathrm{~h} 00-17 \mathrm{~h} 00$
samedi $9 \mathrm{~h} 00-11 \mathrm{~h} 00$
fermeture annuelle entre Noël et le 1 er Janvier

Le service des Archives municipales de Laval a été créé en 1995. Dans l'attente de l'aménagement de locaux plus adaptés, l'essentiel du fonds moderne antérieur à 1940 est encore déposé aux Archives départementales de la Mayenne. 


\section{1) Enrichissement des fonds}

En 2004, les fonds publics se sont enrichis d'une vingtaine de mètres linéaires d'archives, essentiellement liées à l'activité du Centre communal d'action sociale.

10 cartes postales représentant diverses vues de la ville au début du $\mathrm{XX}^{\mathrm{e}}$ siècle ont été acquises et reçues. Plusieurs photos et cartes postales de collections privées concernant le Palais de l'Industrie ont été numérisées ainsi que 171 plans du fonds municipal (architecture et urbanisme).

\section{2) Instruments de recherche et publications}

La base de données TUGAL contient actuellement 6543 fiches relatives aux fonds d'archives et 1458 relatives aux articles de périodiques historiques. L'inventaire des ouvrages provenant du Fonds Laurain-Portemer (ouvrage d'histoire locale) a été réalisé. Il est intégré à l'inventaire informatisé de la bibliothèque du service. Les titres de délibérations du conseil municipal de la période 1952-1961 ont été répertoriés et indexés.

\section{3) Échos et nouvelles}

Le service a participé aux journées du patrimoine en présentant une exposition sur le Palais de l'Industrie. Le résultat des recherches sur l'histoire de ce bâtiment a été présenté lors d'une conférence et sera publié en 2005.

\section{Archives départementales de la Sarthe}

\begin{tabular}{c} 
Fiche signalétique \\
Archives départementales de la Sarthe \\
1 rue Christian-Pineau - 72016 LE MANS CEDEX 2 \\
Téléphone : 02 4354 74 74 - Télécopie : 02 4354 7446 \\
e-mail : archives@sarthe.com \\
site internet : www.archives.sarthe.com \\
Heures d'ouvertures : lundi-vendredi 8h $30-17 \mathrm{~h} 30$ \\
samedi $8 \mathrm{~h} 30-13 \mathrm{~h}$ 00 (selon calendrier) \\
fermeture annuelle la $2^{\mathrm{e}}$ quinzaine de septembre \\
\hline
\end{tabular}

\section{1) Enrichissement des collections}

\section{Archives publiques}

Assoupie au premier semestre 2004, la collecte des archives publiques a repris pour se stabiliser à un peu moins de 300 mètres linéaires $(290,80$ m linéaires), s'orientant plus particulièrement autour des services du conseil 
général, et de deux priorités conjoncturelles : les services déconcentrés de l'État (149,55 m linéaires) et les études notariales (95 m linéaires), très demandeuses. Onze communes, dont une de plus de 2000 habitants, ont en outre déposé leurs archives centenaires (33,10 m linéaires).

\section{Archives privées}

Moins importante qu'en 2003, la prospection d'archives privées s'est cependant poursuivie, avec l'entrée de fonds variés (10,50 m linéaires), parmi lesquels il faut noter :

Archives d'association

- $92 \mathrm{~J}$ : Archives de l'association "Les Amis de Tristan Klingsor" Archives d'érudits

- $18 \mathrm{~J}$ : Complément du fonds Paul Cordonnier-Détrie Archives familiales

- $70 \mathrm{~J}$ : Pièces du chartrier de Maleffre

\section{2) Instruments de recherche et publications}

Les classements, plus modestes que l'année précédente, faute d'effectifs, restent toutefois honorables, puisque ce sont près de 400 mètres linéaires qui ont été traités, représentant quelques 458 pages d'inventaires dactylographiées.

\section{Séries modernes}

Parmi les fonds inventoriés, on signalera la poursuite et l'achèvement du classement de quatre fonds de justices de paix (Bouloire, Château-duLoir, La Flèche et Vibraye).

\section{Série $W$}

Seize versements administratifs ont été traités en 2004. Si l'inventaire des archives du Cabinet de la Préfecture pour la Seconde Guerre mondiale (2000 W) suit son cours, on retiendra également le classement des fonds du commissariat de La Flèche (1445 W, 1447 W et 1448 W), de la trésorerie générale (1286 W) et de l'Office national des anciens combattants (1449 W).

\section{Série J}

Des différents fonds analysés, sont à signaler ceux des Établissements Ciné-Gel (45 J) et du centre éducatif de la communauté Notre-Dame-deCharité dit " du Bon pasteur " $(87 \mathrm{~J})$. 


\section{Archives communales}

Onze fonds d'archives communales déposées ont bénéficié d'un classement. Ce sont celles d'Athenay (260 AC), Aubigné-Racan (255 AC), Brûlon (262 AC), Chemiré-le-Gaudin (68 AC), Courgenard (75 AC), Fyé (263 AC), Neuvillalais (259 AC), Saint-Ouen-de-Mimbré (166 AC), Saint-Pierre-des-Bois (273 AC), Téloché (265 AC) et Vernie (239 AC).

\section{3) Échos et nouvelles}

\section{Numérisation}

Un travail considérable a été réalisé par le biais d'un prestataire externe, avec l'indexation de 175000 images et la numérisation de 350000 pages d'originaux papier (registres d'état civil et listes nominatives) et de 39000 images de microfilms.

\section{Internet}

Le site internet, préparé dans sa version 1 en 2003, a fait l'objet d'une refonte afin de le rendre plus convivial. Très complet, il a été mis en ligne au mois de mai. Parallèlement, a été lancée la préparation de la mise en ligne sur le site de l'ensemble des images numérisées (cadastre, état civil), avec pour objectif de parachever cette opération dès la fin du premier semestre 2005 .

\section{Actions culturelles et éducatives}

Deux expositions ont été montées et présentées par les Archives départementales dans l'année: août);

- La pratique musicale amateur en Sarthe : fin XIXe-début XX $X^{e}$ siècles (mai-

- Des petits dimanches aux fêtes carillonnées : histoire des coiffes de la Sarthe (octobre-décembre).

Plus de 900 personnes, relevant d'associations, de comités d'entreprises..., ont visité les Archives départementales. Quant aux élèves, ce sont plus de 1000 enfants, tant du primaire que du secondaire, qui ont été accueillis dans nos locaux.

Touche finale au nouveau bâtiment et au titre du 1 \% culturel, l'inauguration, au mois d'octobre, de la sculpture de métal "Être noms ", œuvre de Bernard Métais, édifiée à l'entrée du site. 


\title{
Archives Ville Le Mans et Le Mans Métropole
}

\author{
Fiche signalétique \\ Archives municipales et communautaires du Mans \\ 102 rue Gambetta - 72039 LE MANS CEDEX 9 \\ Téléphone : 0243474903 - Télécopie : 0243474368 \\ e-mail : archives@ville-lemans.fr \\ Heures d'ouvertures : lundi-vendredi $8 \mathrm{~h} 30-12 \mathrm{~h} 00$ et $13 \mathrm{~h} 00-17 \mathrm{~h} 00$ \\ (fermeture des portes 15 minutes avant)
}

\section{1) Enrichissement des collections}

\section{Archives publiques}

289 m linéaires de documents (201 m linéaires pour la Ville et $88 \mathrm{~m}$ linéaires pour la CUM) ont été reçus en 2004 tandis que $117 \mathrm{~m}$ linéaires étaient éliminés, soit un accroissement de 172 m linéaires. Parmi les versements les plus notables, nous pouvons signaler :

- Population : registre d'état civil (1904),

- Urbanisme : permis de construire Le Mans (1963-1997), plan d'occupation des sols Le Mans (1988), plan d'occupation des sols d'Arnage (19802000), de La Chapelle-Saint-Aubin (1986-2001), d'Yvré-l'Évêque (1978-1988),

- Département des Ressources Humaines : dossier individuel des agents ayant cessé leurs activités entre 1971 et 2000,

- Communication : animation de fin d'année, commerciale et sportive (1997-2003),

- Services généraux : arrêtés du Maire (1996), arrêtés du Président de la Communauté urbaine du Mans (2002),

- Culture : organisation de manifestations culturelles (1994-2000),

- Voirie : aménagement des rocades du Mans (1970-2001),

- Centre communal d'action sociale : budget et comptabilité (1941-1983),

- Architecture : 4000 calques de bâtiments municipaux et communautaires (1950-1980).

\section{2) Instruments de recherche et publications}

\section{Base de données}

9680 notices ont été saisies et indexées sur le logiciel Avenio qui fait office d'instrument de recherche.

\section{Archives privées}

Traitement et indexation des fonds entrés par voie extraordinaire (travaux de recherche, pièces isolées, dons, dépôts ou achats) et notamment le don de Monsieur Martin portant sur la vie culturelle et sportive de la Ville du Mans (Comité permanent des fêtes de la Ville du Mans, 1935-1989; 
Union sportive du Mans football, 1943-1999; le carnaval d'été en films $11 \mathrm{~mm}$ et $8 \mathrm{~mm}, 1959-1988)$.

\section{3) Échos et nouvelles}

Poursuite de la campagne de restauration du fonds ancien et moderne : 11 registres paroissiaux (1686-1792), registre de recensement de la population (1906), 8 matrices cadastrales (1850-1914), une vingtaine de plans de bâtiments (XVII ${ }^{\mathrm{e}}$-début $\mathrm{XX}$ e siècle), une vingtaine de plans voyers (1848).

Numérisation des registres d'état civil de 1903, des bulletins de salaire 2004, des plans cadastraux (1810-1815), des plans voyers (1814-1877), des plans de ville (XIXe siècle-1946), des plans de bâtiments (1819-1904), des plans de quartiers (1693-1935).

Cadastre et plan voyer : fin de traitement de l'ensemble du fonds, soit 854 planches pour Le Mans, ses villes annexées et des villes membres de la communauté urbaine du Mans ( $\mathrm{XIX}^{\mathrm{e}}-\mathrm{xx} \mathrm{e}^{\mathrm{e}}$ siècle).

Conception et animation d'une émission mensuelle de radio consacrée à des lectures thématiques d'archives.

\section{TOURAINE}

\section{Archives départementales d'Indre-et-Loire}

\section{Fiche signalétique}

Archives départementales d'Indre-et-Loire

Centre des Archives historiques (archives antérieures à 1940, sauf état civil, cadastre et archives communales communiqués sur l'autre site)

6 rue des Ursulines -37000 Tours

Téléphone : 0247608888 - Télécopie : 0247608889

e-mail : archives@cg37.fr

Heures d'ouvertures : lundi-vendredi $9 \mathrm{~h} 00-12 \mathrm{~h} 30$ et $13 \mathrm{~h} 30-17 \mathrm{~h} 00$

Les $1^{\text {er }}$ et $3^{\mathrm{e}}$ samedis du mois, $9 \mathrm{~h} 00-11 \mathrm{~h} 45$

Centre des Archives contemporaines

41 rue Michaël-Faraday -37170 CHAMBRAY-LES-TourS

Téléphone : 0247808900 - Télécopie : 0247808909

Heures d'ouvertures : lundi-vendredi $9 \mathrm{~h} 00-12 \mathrm{~h} 30$ et $13 \mathrm{~h} 30-17 \mathrm{~h} 00$

Les $2^{\mathrm{e}}$ et $4^{\mathrm{e}}$ samedis du mois, $9 \mathrm{~h} 00-11 \mathrm{~h} 45$

Les planches numérisées du cadastre ainsi que les 7800 cartes postales numérisées sont consultables sur les deux sites 


\section{1) Enrichissement des collections}

\section{Archives publiques}

Il convient de noter le versement des dossiers du Cabinet du Préfet couvrant les années 1966-1997, qui enrichit les fonds du site de Chambray pour la période précédant la décentralisation comme pour celle qui la suit.

\section{Archives privées}

Le fonds de Beaumont a été sensiblement enrichi par les plans du marquisat, réalisés au XVIII ${ }^{\mathrm{e}}$ siècle, qui viennent ainsi compléter les précédents dépôts.

Par ailleurs, une collaboration fructueuse avec la commission des archives diocésaines de Tours a permis l'élaboration d'un premier récolement des archives du diocèse; le répertoire est désormais disponible en salle de lecture.

\section{Bibliothèque}

Tous les ouvrages acquis depuis 1990 sont désormais décrits sur le catalogue informatisé de la Bibliothèque départementale de Touraine, consultable à l'adresse [http://www.cg37.fr/lireentouraine/]. Le don d'une importante bibliothèque tourangelle (65 m linéaires) est venu enrichir les collections du service, en particulier pour des ouvrages d'histoire locale.

\section{2) Instruments de recherche}

Le classement des archives de la colonie agricole et pénitentiaire de Mettray (fonds $114 \mathrm{~J}$ ) a donné lieu à la publication d'un instrument de recherche complet et à l'organisation, par le service, d'un colloque consacré à cet établissement. Les actes du colloque paraîtront aux Presses Universitaires de Rennes à l'automne 2005.

Pour mémoire, près de 7800 cartes postales sont accessibles en ligne par le biais du site du conseil général d'Indre-et-Loire [www.cg37.fr] rubrique "Les sites du conseil général, Cartes postales de Touraine ".

\section{3) Échos et nouvelles}

L'envoi de bobines de microfilms des registres paroissiaux et d'état civil a repris vers les autres services départementaux d'archives, à partir du site de Chambray.

Le cours de paléographie médiévale de l'Université de Tours est désormais assuré par le directeur du service.

Le projet de Maison de la Mémoire à Maillé poursuit son chemin, avec le choix de l'architecte et du scénographe. L'ouverture en est prévue à la fin de 2005 . 


\section{Archives municipales de Tours}

\begin{tabular}{|c|}
\hline Fiche signalétique \\
Mairie de Tours, $1-3$ rue des Minimes, BP $3215-37032$ TOURS CEDEX 1 \\
Section historique : place Saint-Éloi \\
Heures d'ouvertures : lundi-vendredi $8 \mathrm{~h} 45-12 \mathrm{~h} 00$ et $13 \mathrm{~h} 45-17 \mathrm{~h} 15$ \\
Téléphone : 0247216181 - Télécopie : 0247390610 \\
Section contemporaine : Mairie de Tours, $2^{\mathrm{e}}$ sous-sol \\
Téléphone Archives : 0247216954 - Télécopie $: 0247216936$ \\
Téléphone Photothèque : 0247216953 \\
e-mail : dadp@ville-tours.fr \\
site internet : www.tours.fr (rubrique Culture) \\
Heures d'ouvertures : lundi-vendredi $9 \mathrm{~h} 00-12 \mathrm{~h} 00$
\end{tabular}

Les Archives municipales sont installées sur deux sites depuis 1990, et couvrent $5,5 \mathrm{~km}$ de rayonnages.

La section historique (place Saint-Éloi) conserve les séries anciennes : entre autres une charte de 1035 environ, une très belle série de registres de comptes depuis 1358, les délibérations municipales depuis 1408, et les séries modernes, notamment les dossiers de la fusion avec la commune de Saint-Étienne en 1845, et tous les documents jusqu'en 1945. Y sont aussi consultables les archives privées, comme celles du maire de la Libération, M. Jean Meunier, ou de la famille de Clocheville, dont l'hôpital pour enfants porte le nom.

La section contemporaine (au $2^{\mathrm{e}}$ sous-sol de la mairie) conserve pour sa part les documents de 1945 à nos jours, en particulier les plans de la reconstruction de la ville dans l'immédiat après-guerre et les dossiers de la mise en place du Secteur sauvegardé.

La photothèque, créée en 1985 , également située au $2^{\mathrm{e}}$ sous-sol de la mairie, est riche de plus de 100000 clichés et vient encore de s'étoffer avec l'achat du fonds du photographe tourangeau Jean Bourgeois (près de 30000 clichés). Elle est complétée par une série de 4000 cartes postales anciennes.

\section{1) Enrichissement des collections}

\section{Archives publiques}

Engagée l'an passé, la contraction du volume des versements administratifs, notamment par une meilleure préparation dans les services, s'est concrétisée en 2004 par une arrivée de 137 m linéaires, contre plus de $300 \mathrm{~m}$ linéaires précédemment. Associée à une intense politique d'élimination, 260 m linéaires en 2005, elle permet une stabilisation de la gestion des magasins, saturés depuis quelques années. Le service a connu un enrichissement particulier par le versement de trois maquettes : l'hôtel de ville 
de Tours (1980), l'hôtel de ville de Saint-Symphorien (1937) et le projet d'urbanisme de Tours Nord (1965).

\section{Archives privées}

Les Archives municipales de Tours ont connu en 2004 le don d'un fonds documentaire sur l'histoire de la médecine à Tours. Réuni par le docteur Jean Moline qui a mené d'importantes recherches sur la Société médicale de Tours fondée en 1801, ce fonds est constitué de notes de recherches, de quelques pièces d'archives, mais surtout d'une importante bibliothèque sur l'histoire de la médecine et de la pharmacie.

Par ailleurs, le service s'est enrichi par le complément du fonds Clavier (1500 reportages de photographies de journalisme), par l'arrivée du fonds Odile Rousseau (artiste contemporain ayant réalisé des dessins sur l'architecture de Tours), du fonds Guettier de Percin (pièces isolées sur la construction de l'hôtel de ville), du dossier familial sur le résistant André Champault, d'un dossier de coupures de presse sur la guerre 1939-1945 et enfin d'un dossier sur la famille Benoit (ancien directeur du cimetière).

\section{Archives audiovisuelles}

Gérée par les Archives municipales, la photothèque municipale s'est enrichie en 2004 de 9544 clichés, archivés principalement par le Service de la Communication. Mais cette section a bénéficié d'un don important : les archives photographiques du studio Germain. Composé de 2300 reportages, ce fonds contient principalement des portraits, mais aussi de précieux témoignages des manifestations culturelles tourangelles. Il couvre la période 1948-2002.

\section{2) Instruments de recherche et publications}

Le classement des séries de la période moderne (1790-1945) se poursuit et est achevé pour les séries $1 \mathrm{O}$ (voirie urbaine) et $3 \mathrm{O}$ (navigation et régime des eaux) qui sont maintenant dotées d'un répertoire dactylographié. En section contemporaine, le classement définitif de la reconstruction (1940-1965) a aussi abouti à la réalisation d'un instrument de recherche dactylographié.

En 2004, le service éducatif des Archives municipales a édité un guide d'exploitation de l'exposition " Tours, 1000 ans d'identité communale ". Par ailleurs, en collaboration avec l'IUFM d'Orléans-Tours, deux dossiers pédagogiques ont été publiés, l'un sur les "Écoles d'autrefois - 1789-1945 ", l'autre sur « Patrimoine et archives : travaux publics, industries, ponts, voirie du XVI ${ }^{\mathrm{e}}$ au XIX ${ }^{\mathrm{e}}$ siècle". 


\section{3) Échos et nouvelles}

Dans le domaine de la diffusion, les Archives municipales ont multiplié les opérations, notamment par des expositions variées. Ainsi, le service a accueilli et enrichi les réalisations d'établissements extérieurs :

- L'hygiène au Moyen Âge

- 100 ans, 100 métiers (le personnel et le centenaire de l'hôtel de ville)

- Le lycée Balzac a 100 ans

Il a par ailleurs produit des expositions :

- Le vigneron et sa cave (fête des vins "Vitiloire ")

- Tours libérée (soixantenaire de la Libération)

- Mémoire cheminote (rétrospective des anciens ateliers du chemin de fer)

- Tours et ses hôtels de ville (centenaire de l'hôtel de ville)

Cheville ouvrière des manifestations du centenaire de l'hôtel de ville, les Archives municipales ont aussi accompagné le développement du programme éducatif de la ville sur l'approche de la citoyenneté qui s'est traduit par la fréquentation de 1775 élèves aux ateliers du service éducatif en 2004. 


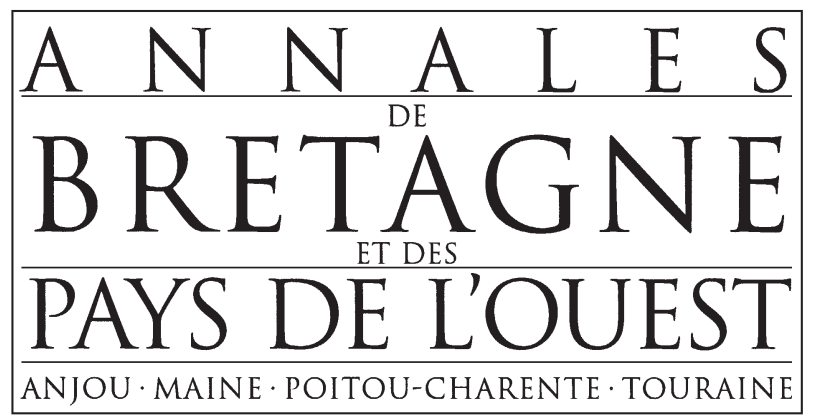

\section{ABONNEMENT}

FRANCE, 4 numéros : $32 €$-ÉTRANGER, 4 numéros : $40 €$ (Franco de port)

ADRESSE D'EXPEDITION

(pour toute commande)

Nom

Prénom

Organisme

Adresse

Code postal

Ville

Pays

Ce bon de commande accompagné d'un chèque à l'ordre de Agent comptable de l'Université Rennes 2 est à envoyer à l'adresse suivante :

Presses Universitaires DE RenNes

Campus de La Harpe

2 rue du Doyen-Denis-Leroy

35044 RENNES Cedex

FRANCE 


\section{QUELQUES REGLES DE PRESENTATION DES TEXTES A L'USAGE DES AUTEURS}

1 - Indiquer sous le titre de l'article la qualité et l'adresse institutionnelle de l'auteur Ex. : Xxxx Xxxxxx, maître de conférences en histoire contemporaine, université de X., groupe de recherches de rattachement.

2 - Remplacer le plus possible les majuscules (sauf pour les lettres initiales des noms propres) par des petites capitales. Ceci dans les titres, les sigles, les noms d'auteurs... Indiquer aussi les siècles en petites capitales : $\mathrm{XV}^{\mathrm{e}}$ siècle et non XVe siècle.

Accentuer les majuscules.

3 - Abréviations : en user le moins possible

Pas d'abréviations pour le nom des revues, surtout pour les revues locales qui ne sont connues que des locaux.

Pour les dépôts d'archives :

- ne pas mettre ADIV ou AD 35 mais : Arch. dép. d'Ille-et-Vilaine, du Morbihan...;

- préférer Arch. nat. à AN;

- mettre les noms des bibliothèques en entier.

Développer les sigles entre parenthèses lors de leur première occurrence.

\section{4 - Références bibliographiques}

- mettre toujours et partout les prénoms et les noms (ne pas abréger le prénom par une initiale);

- utiliser les petites capitales pour le nom propre et non les majuscules, utiliser les minuscules pour le prénom; les séparer par des virgules.

Nom, Prénom, Titre de l'ouvrage en italique, Lieu d'édition, éditeur, "collection " (éventuellement), année, pages.

Une bibliographie peut être donnée en fin d'article si cela est nécessaire.

\section{5 - Forme des notes}

Préférer la forme traditionnelle (référence biblio. développée lors de sa première occurrence, abrégée ensuite) à la forme anglo-saxonne (nom, date, page).

\section{6 - Titres et sous-titres}

Il est préférable de s'en tenir à deux niveaux. Mais si cela s'avère nécessaire afin de ne pas obtenir de trop longues pages trop compactes, un troisième niveau d'intertitres peut être utilisé.

\section{7 - Cartes et documents photographiques}

- Les cartes au trait devront être d'une EXCELLENTE QUALITE GRAPHIQUE sans collage hasardeux ni légende manuscrite.

- Il est toujours préférable de FOURNIR LES ORIGINAUX, sinon, un fichier informatique au format .EPS pour les cartes et plans ou .TIF pour les photographies. en aucun cas les images seront intégrées au fichier word seulement : IL FAUT EGALEMENT LE FICHIER SOURCE.

8 - Autres questions non évoquées ici...

consulter la rédaction à l'adresse ci-dessous.

9 - Donnez une adresse mail et téléphone où l'on puisse vous joindre facilement au moment où l'on fait la mise en page de votre article.

\section{Contact}

Annie Antorne, Secrétaire de l'Association pour la publication des $A B P O$ université de Rennes 2, Département Histoire, Place du Doyen-Henri-Le-Moal - 35043 RenNes Cedex e-mail : annie.antoine@uhb.fr

Les manuscrits sont à envoyer à l'adresse ci-dessus

Ouvrage achevé d'imprimer

sur les presses du service reprographique

de l'Université Rennes 2 Haute-Bretagne

en décembre 2005

Imprimé en France 